\title{
The Role of Surface Chemistry in Specimen Preparation for Structure-Function Determination of Nanomaterials and Biological Systems.
}

\author{
John M. Miller, Janet Teshima, and Daniel Moore \\ Dune Sciences, Inc. 1900 Millrace Drive, Eugene, OR 97403
}

The understanding of structure-function properties in nanomaterials and in biological systems is paramount for the development of next-generation nano-enabled processes, devices and applications; new drugs and medical devices; and in the assessment of the environmental and health risks of nanomaterials. Central to this understanding is the need for correlating accurate metrology from a variety of analytical methods that can probe both structure and function. The key to metrology is sample preparation that is part of the experiment design and does not change or distort the sample.

Today's advanced instrumentation for optical and electron microscopy, surface analysis and spectroscopy provides unmatched opportunities to interrogate the structure and chemistry of materials on the molecular and nano-scale. However, the importance of specimen preparation to ensure representative and accurate results is often overlooked as a primary consideration in the design of experiments. In fact, specimen preparation often imparts artifacts that can bias interpretation of data and convolute the correlation of structure-function properties. Many analytical methods require the use of surrogate or otherwise processed samples for analysis that can adversely affect accurate metrology. For example, dissolved or dispersed samples that are prepared for analysis using SEM or TEM are often drop cast directly onto a substrate surface. The approach frequently results in aggregation of particles during drying, poor control over the distribution of material on the substrate, and is not representative of the sample. Aggregation effects can also be observed in biological samples. These effects reduce spatial resolution, increase analysis time and can skew the interpretation of particle size/structure/composition. Moreover, current sample preparation is often an art that results in significant variability in sample quality.

The current study investigates the role that surface chemistry can play to improve the quality and reproducibility of specimen preparation and facilitate accurate correlative analysis of nanomaterials and biomolecules. The surface properties (composition, uniformity, charge) dictate the performance of many materials and biological systems on the nanoscale. These surface properties can be exploited to enhance sample preparation by tuning the surface chemistry of the specimen substrates to promote affinity towards a wide variety of different materials as shown in Fig. 1. We explored two general approaches for sample preparation: 1) electrostatic, non-specific assembly of materials, and 2) covalent attachment of nanomaterials. In this study, SMART Grids ${ }^{\mathrm{TM}}$ functionalized with five different surface chemistries were used to promote assembly of ligand stabilized Au nanoparticles and adeno-associated virus (AAV2) particles as model systems. SMART Grids are functionalized TEM grids that utilize electron transparent $\mathrm{SiO}_{2}$ membrane windows. The hydrophilic, ultraflat surface of the SMART grids provides and ideal platform for multi-technique analysis that allows for direct correlation of structure and function data from the same sample. TEM samples were prepared using a variety of gold nanoparticles with sizes ranging from $1.5 \mathrm{~nm}$ to $60 \mathrm{~nm}$ and surface chemistries that 
represent positive, neutral and negative surface charge. As shown in Figure 2, there is a significant dependence on sample quality when the substrate is surface-matched to the analyte of interest. Negatively charged Au nanoparticles showed a strong affinity for the positively charged SMART Grid and resulted in a high concentration of isolated nanoparticles and reduced sample preparation time to $<5$ minutes. Positively charged particles showed a similar affinity to negatively charged grids. Control samples for non-functionalized grids resulted in significant

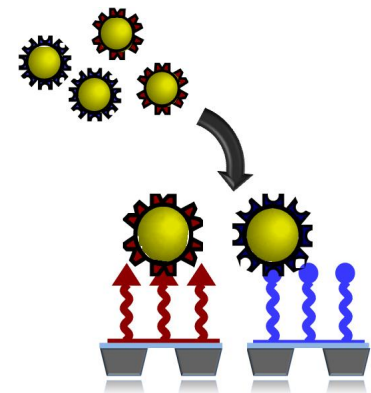

Fig. 1 Schematic of surfacechemistry enhanced sample preparation for nanoscale characterization. aggregation of the nanoparticles resulting from drying.

The covalent attachment of materials directly to the SMART Grids surface can be achieved either through direct linkage or through a linker molecule. Once attached, the materials can be further processed, purified, or analyzed without concern for sample aging. The covalent tethering of materials also creates new opportunities for in-situ probing of interfacial relationships in biological systems, in the sequential analysis of materials under a range of conditions, and to assess the toxicology and environmental fate of nanoparticles. Figure 3 shows a TEM micrograph of AAV2 stained with uranyl acetate covalently attached to the chemistry can play in standardizing sample preparation methodologies and allow for accurate correlative metrology.
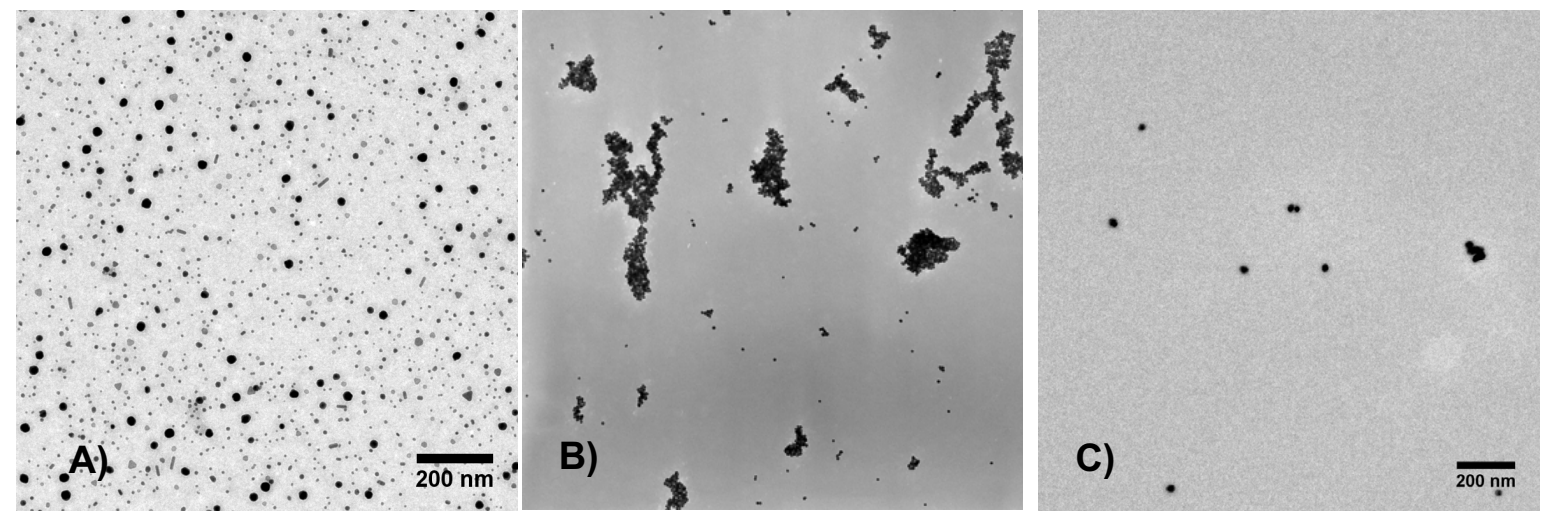

Figure 2. Influence of surface chemistry on distribution and concentration of negatively-charged gold nanoparticles deposited on a) Positively charged SMART Grid, b) non-functionalized SMART Grids, and C) commercial SiOx grid. TEM images taken at CAMCOR, University of Oregon.

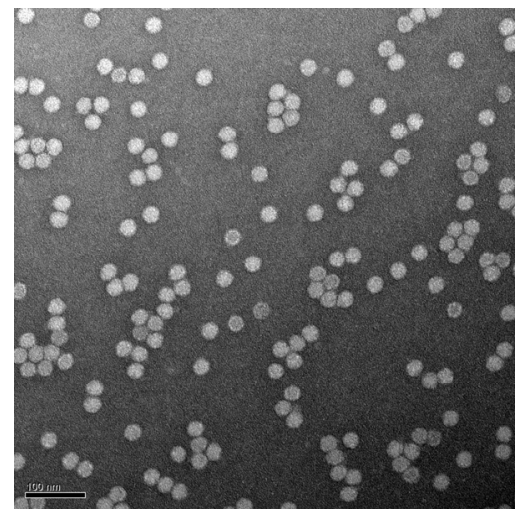

Figure 3. AAV2 particles covalently attached to SMART Grids surface.

Sample courtesy of Dustin McGraw, Oregon Health Sciences University. 